\title{
Kinematics Parallel Mechanisms Design Particularities Focused on Additive Manufacturing
}

\author{
Diego A Nunez*, Sebastian Gonzalez, Juan Guacheta, Mauricio Mauledoux and Oscar Aviles
}

Davinci Research Group, Mechatronics Engineering, Universidad Militar Nueva Granada, Carrera 11 101 80, Bogota, Colombia

Received 18 August 2021; Accepted 30 September 2021

\begin{abstract}
Additive manufacturing has had a relevant role in the new industrial revolution owing to the way of a part are designed, manufactured, and distributed to final consumers. Current commercial mechanisms have problems related to their limited degree of freedom. In order to improve them, there are possibilities to adapt either a serial manipulator or a kinematics parallel mechanism to the process. The first ones have disadvantages related to cost, inertia ant answer time; the second ones can solve these issues. However, using these kinds of mechanisms is necessary, considering other types of aspects such as mechanical configuration, dynamics behavior, and workspace. For these reasons, this work collects information related to the above features of parallel mechanisms focused on additive manufacturing. Finally, this kind of analysis and information can be useful for other processes or studies that use kinematics parallel mechanisms.
\end{abstract}

Keywords: Parallel mechanisms, additive manufacturing, workspace, dynamics, kinematics.

\section{Introduction}

Among options of multidirectional adding, there are industrial serial robotic arms and parallel mechanisms (PMs). The first ones have demonstrated precision and structural stiffness; however, the cost of these devices is high, and they have a weak response to high-speed requirements due to these kinds of movements cause vibrations, damping, inertial increment, and low responses for perturbations [1], [2]. PMs are efficient positioning mechanisms because of their dynamics, precision, and structural stiffness due to their close loops kinematics. Nevertheless, their dynamics and control analysis is complex, and their workspace is much less than its counterpart [3].

Over the last decade, more than 200 different PMs have been developed [4]. The majority have been focused on automatization, pick and place tasks, and simulators because these works require high speed and acceleration machines. Since Stewart's work in 1965, PMs have proved that they complement serial robots because they bear higher loads; however, despite many research efforts and previous claims, the industry does not technologically adopt these PMs yet, and many of these are just applied in laboratories [5].

Additive manufacturing (AM) has revolutionized the design, manufacturing, and distribution of products due to the speed of proliferation and variety of brands. Nevertheless, there is a lack of comprehension about de design principles, manufacturing guides, standardization, and mechanical improvements [6].

Considering their mechanical advantages, PMs can be implemented in AM and may use rigid or cabled guided arms. Cable-guided PMs are used in applications that require high WSs. Admittedly, their limitations are based on they just work if cables are stressed; thus, they are susceptible to uncontrollable pendulum movements when the platform moves at high speed, or there are adverse environmental conditions. Additionally, a guided cable system with j DOF (DOF) requires a $\mathrm{j}+1$ actuator [7]. Which is another disadvantage compared to rigid MPs, which normally use the same number of actuators as the degrees of freedom of the movable plate [8]. Hence, rigid arms PMs which usually use the same number of actuators and DOF, are better options for AM.

Technological challenges of PMs related to AM consists on: mechanical design, dynamics analysis, and workspace study, among others. Therefore, it is necessary to consider the features mentioned above to implement PMs in AM, which is the aim of this text.

\section{Mechanical Configuration}

Many PMs have been developed, from hexapod type advances such as Stewart's, which were initially rejected due to their control's difficulty, to more popular designs such as Delta type.

Previously, the importance of rigid arms PMs was mentioned, and most of them have a movable platform where the dispenser or material curing system can be fixed. There are some examples of PM which may be interestingly applied to AM process.

Fu et al. [9] presented a robot consisting of 6 DOF, which had three identical arms connected from the fixed base plate to the movable plate. Each of them has one active rotational joint, two passive rotational joints, one active prismatic joint, and one universal type. The 6 active joints are actuated by servo motors and sensed by linear potentiometers (Fig. 1). The advantage of this robot is that the translations and rotations were decoupled; the end position of the movable plate depends only on the prismatic actuators, while the final rotation depends on the 6 actuators. The reconfiguration of the structure is simplified due to the modular design. 


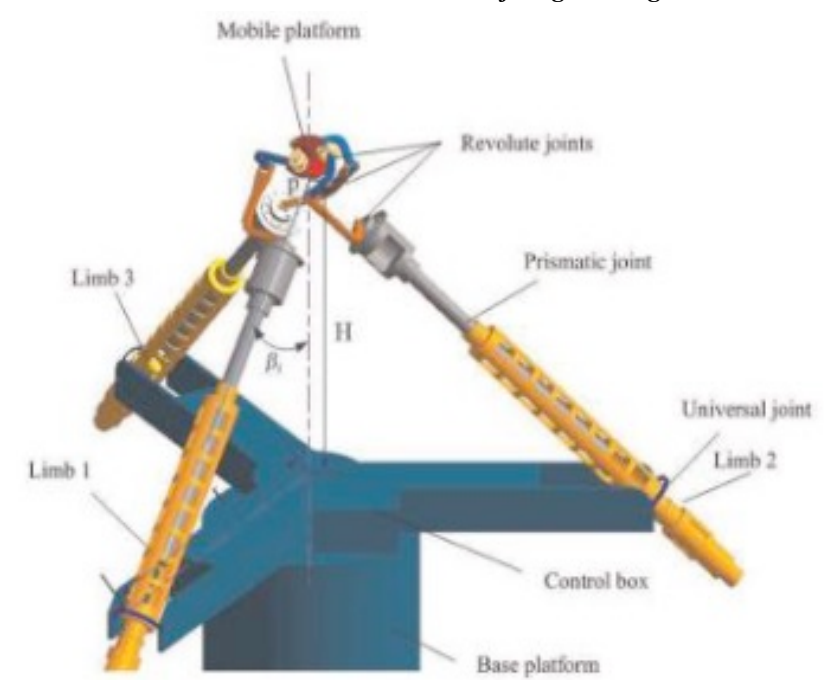

Fig. 1. 6 DOF PM. [9]

$\mathrm{Li}$ and Angeles [10] designed a robust 6 DOF parallel robot type 3-CCC (Fig. 2) whose architecture is ahead of other parallel configurations. It's C-drive type actuator provided higher stiffness, load-carrying capacity and diminished the inertia effects.

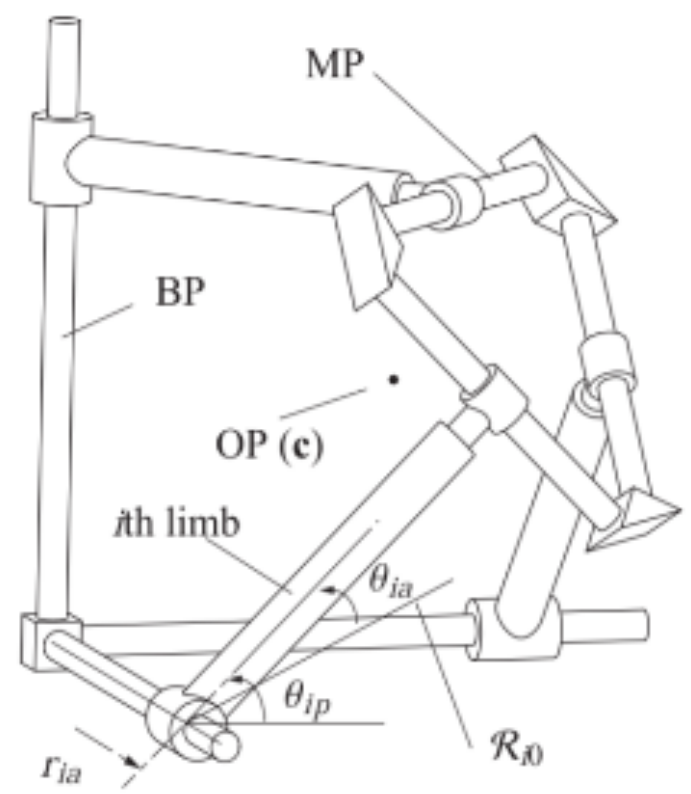

Fig. 2. 6 DOF PM with 3 CCC arms [10].

In some circumstances, when the dispenser or nozzle has a considerable weight, PMs, in which its arms support one or more motors, is not recommended because the location of the motors at the extremities increases system inertia, affecting the precision and rigidness. For this reason, there are PM prototypes with the actuators on the base.

Wei Ye et al. [11] presented a reconfigurable parallel mechanism focused on the extrusion process of fused material, seeking that it could reconfigure its structure according to the various task. Accordingly, they created a parallel manipulator that can translate their mobile platform on the $\mathrm{X}, \mathrm{Y}$, and $\mathrm{Z}$ axes and rotate it on the $\mathrm{X}$ and $\mathrm{Y}$ axes, allowing to add material on flat, cylindrical, circular, and freeform surfaces. Because they required a minimum of 3 DOF, it was necessary by design to make the mobile platform join the fixed base employing three arms. Two of them were identical and had a PMM-URR-type kinematic characteristic (PMM represents a planar metamorphic mechanism. U represents a universal joint or joint, and $\mathrm{R}$ represents a rotational joint). The use of planar metamorphic shape gives the arms the characteristic of being reconfigurable hybrids. This type of component provided 6 DOFs. The configuration proposed in this design, due to the third RRUR type arm, restricted DOFs and configured the robot to four modes of operation. i.e., three translations, three translations and only rotation in $\mathrm{X}$, three translations and just one rotation in $\mathrm{Y}$, and three translations and rotations allowed in $\mathrm{X}$ and $\mathrm{Y}$ (Fig. 3).

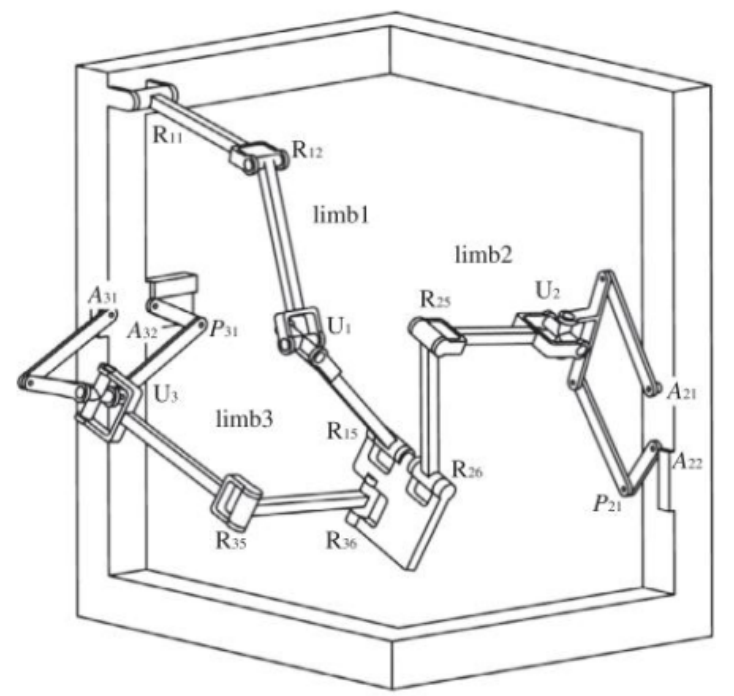

Fig. 3. Reconfigurable PM [11].

Due to the complexities and costs of manufacturing PMs, Song et al. [12] designed and manufactured a low-cost robot focused on fused deposition material (FDM). The reasons for selecting this technique were the vibrations, and axial loads involved in the AM process compared to other methods such as chip removal are lower. The machine was based on a Gough-Stewart-type platform. The movement of the linear actuators was generated with an adaptation of the motors to ball screws guided by smooth shafts. 6 motors were used, and the height control of the material deposition was sensed with a laser and a camera. The camera recorded the laser redpoint, and the relation between laser-point distance from the center of the camera and the distance between the nozzle helped to determine the surface.

Dutta et al. [13] presented a 6 DOF RSS parallel robot (Fig. 4) with a new detection method and compliance to an external force applied on the mobile platform, avoiding external force sensors. This development reduces cost, generates more compact PMs, and makes them more versatile.

On some occasions, one requires machines capable of generating higher speeds and accelerations. So, Filho and Cabral [14] worked on a Hexa PM whose fixed plate was connected to a movable plate by equal arms with rotational, universal, and spherical (RUS) joints. The arms were located in a circular pattern centered in the base plate contributing 6 DOFs (Fig. 5); this configuration added broad movement in the vertical axis ideal for large printings. 


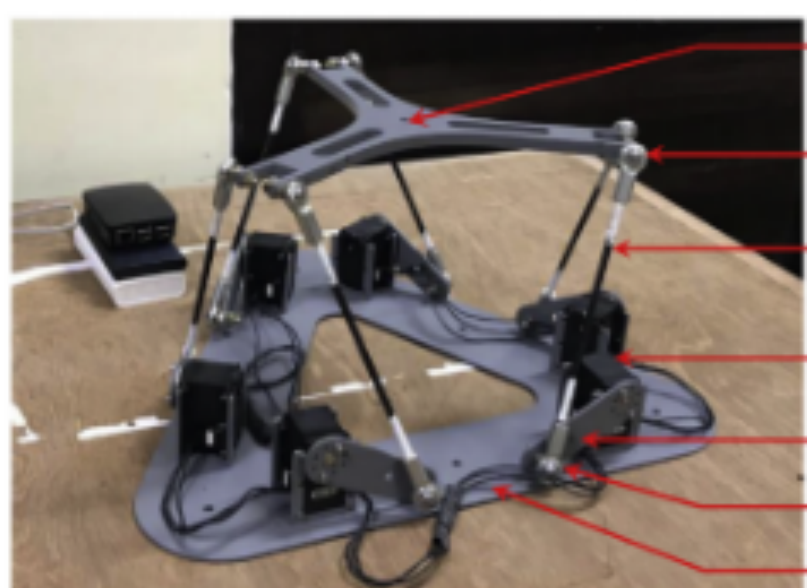

Fig. 4. 6 DOF RSS PM [13].

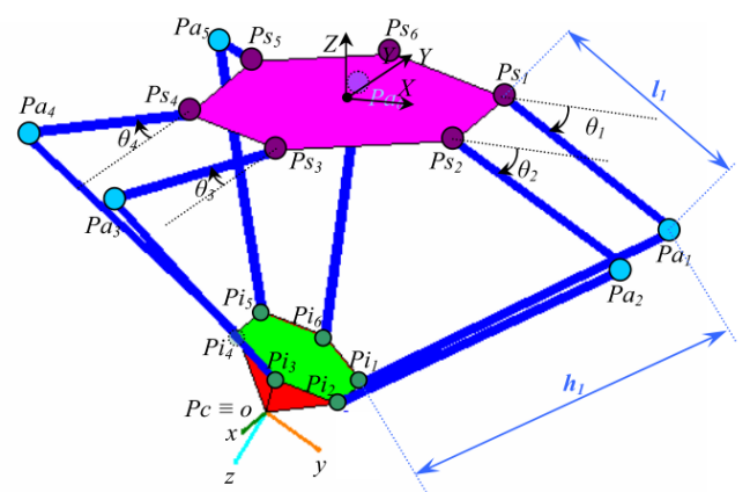

Fig. 5. 6 DOF RUS PM [14] ${ }_{D_{2}}$

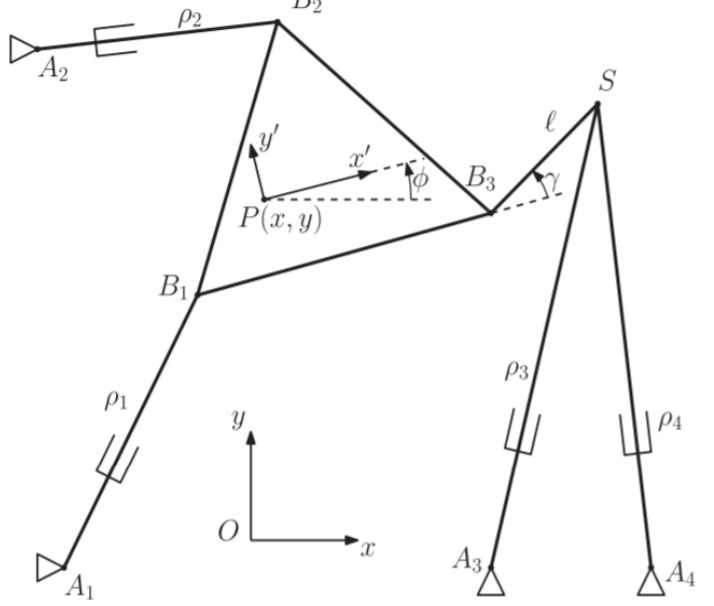

Fig 6. Geometric model of the $2 \mathrm{PR}$ mechanism

Some PMs allow only a rotational range of motion at specific points due to mechanical singularities. Some authors have solved that by adding an extra arm that joins the base with the movable plate. Gosselin et al. [15] and Schreiber and Gosselin [16] proposed and analyzed 4 DOF PMs composed of four active prismatic arms. At the end of the two arms, there is a rotational joint attached to the fixed base. At the other end of each is a rotational joint attached to the movable plate. The other two arms at one end are each attached to the fixed base; at the other end, both arms are connected with a spherical joint. Between the spherical joint and the movable plate, there is an arm, the connection between this arm and the movable plate is through a rotational joint (Fig. 6). This configuration allowed the free rotation of the movable plate without any restriction, but there was redundancy, antagonistic forces, and an increase in the difficulty of movement control.

At other times, more torque is required in the extremities, so more joints must be activated. Gayral et al. [17] focused on a three-armed compound PM with increased activity in the rotational joints. The researchers proposed a $6 \mathrm{DOF}$ parallel robot based on three RRP articulated arms with two active joints and a transverse prismatic joint. A rod connecting the movable arms with the final triangular actuator can slide. The bars are attached to the final actuator using universal joints located at the vertices (Fig. 7). The added value of the design is that the actuators are located at the base of the robot. The location of the actuators is due to the use of a two DOF planetary pulley system, which helps transmit motor power from the base to the active rotational joint. Because the motors are in the base of the robot, the extremities' mass and inertia are reduced, generating a light manipulator. The robot requires 6 motors for its operation.

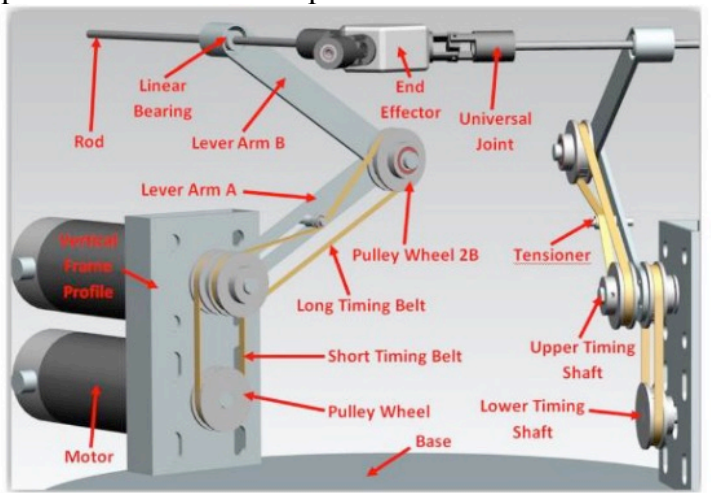

Fig. 7. 6 DOF RRP PM [17].

One of the most used PMs has been the Delta type, which offers three DOF in a light structure. However, based on its design, some prototypes have been made, increasing its DOF and workspace (WS). Li and Angeles [18] proposed a robot based on the distribution of a Delta robot, with three arms that join the movable plate to the base. The arms have a CPS-type kinematic distribution where the actuator is in the cylindrical joint. The cylindrical actuator consists of its interior of a RHHR type mechanism. Two screws with opposite threads, one in front of the other, aligned axially and parallel to the base and united by the helical joint (Fig. 8). Depending on the direction and speed of rotation, the cylindrical joint rotates or moves, determining the robot with 6 DOF with actuators close to the base, avoiding inertia, and increasing the WS with respect to the Delta type robot.

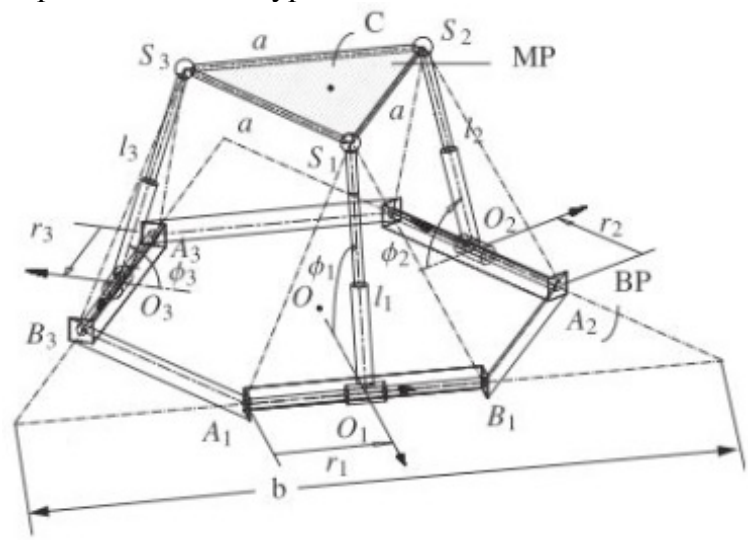

Fig. 8. CPS arms PM [18]. 
Another modification of a Delta type robot was proposed by Azulay et al. [19]. They created a parallel robot that offers 6 DOF supported on a structure where the movable plate is attached to the base by three arms. Each of the arms has the following characteristics evidenced in Fig. 9 There is a prismatic type actuator at the junction between the arm and the base that moves circularly to the $\mathrm{Z}$ axis. There is a second prismatic actuator that moves in a normal direction with respect to the $\mathrm{Z}$ axis on this actuator. Above the last actuator, there is a third cylindrical joint attached to the arm and allows its rotation. At the other end of the arm, there is a spherical joint attached to the movable plate.

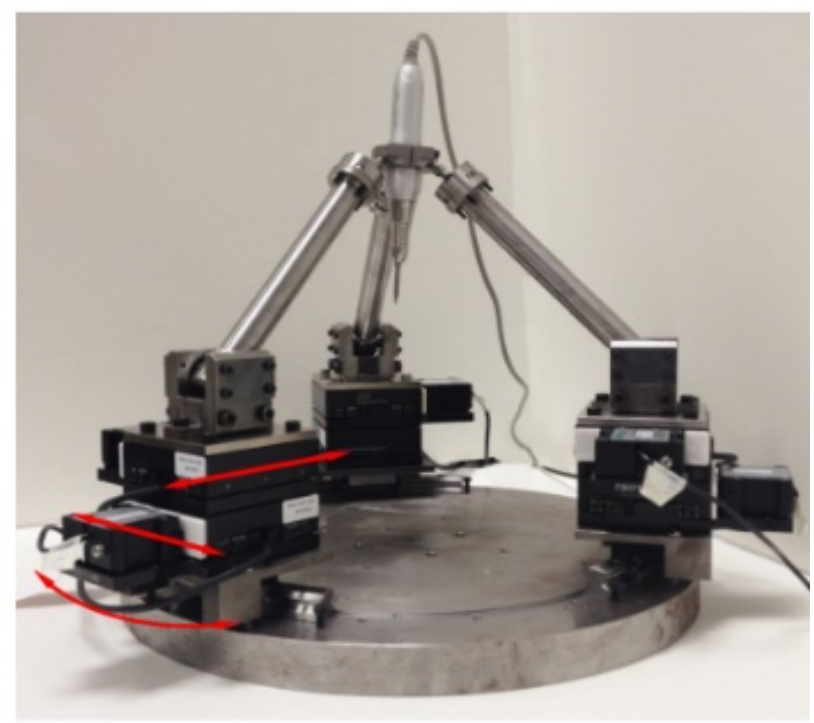

Fig. 9. Azulayb 6 DOF PM [19].

The normal movement of the base's prismatic actuators increases the WS in the $\mathrm{X}$ and $\mathrm{Y}$ axes, so this configuration has been proposed for high precision machining and AM. Some works have also been done on manipulators with decoupled kinematics. An example is the development of Song et al. [20], who proposed a design strategy for robots with 4-6 DOF. For this, they provide the translation in $\mathrm{X}$ and $\mathrm{Y}$ through a platform with prismatic joints. On this platform, they initially propose an actuator platform supported by three UPS -type kinematics chains and in the center one UP type. The actuator platform is rotated $90^{\circ}$ with respect to the Yaxis (Fig. 10). Within the proposed methodology, the configuration to increase or decrease DOF is explained. According to the aforementioned study, Sun and Lian [21] optimized this PM with a multiobjective approach resulting in a Pareto front of stiffness versus mass. This technique may be implemented in other PMs.

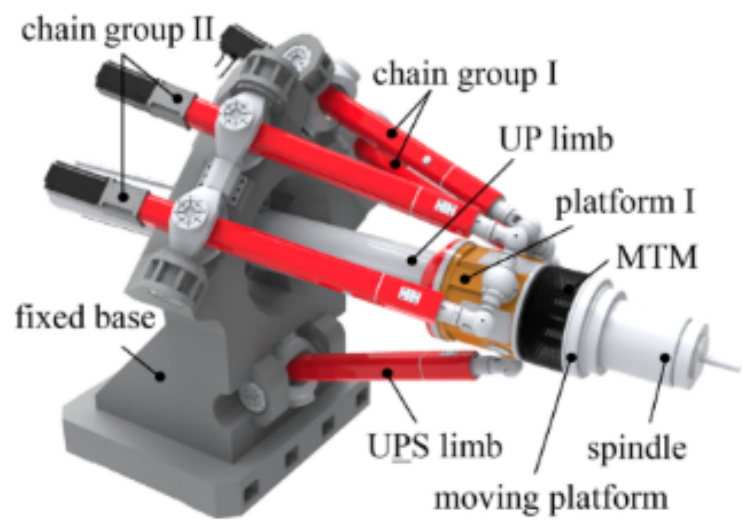

Fig. 10. Song 5 DOF PM [20].
Another work on manipulators with decoupled kinematics was worked by Zhang et al. [22]. The PM is based on a flat robot with 5 DOF (Fig. 11). The structure has a vertically aligned plane manipulator with all its movements on that plane. The planar mechanism has four identical PRR-type arms, an intermediate arm, and a movable plate. The movable plate is attached to one end of two arms by rotational joints. At the other end, each one of the rotational joints of each arm is united to a prismatic joint which is collinear. The ends of the two remaining arms are rotationally attached to the intermediate arm, which at its other end is rotationally attached to the movable plate. Similar the previous two arms, at the other end, there is a rotational joint between the arm and collinear prismatic joints. This PM provides vertical and rotational translational movements in the $\mathrm{X}$-axis.

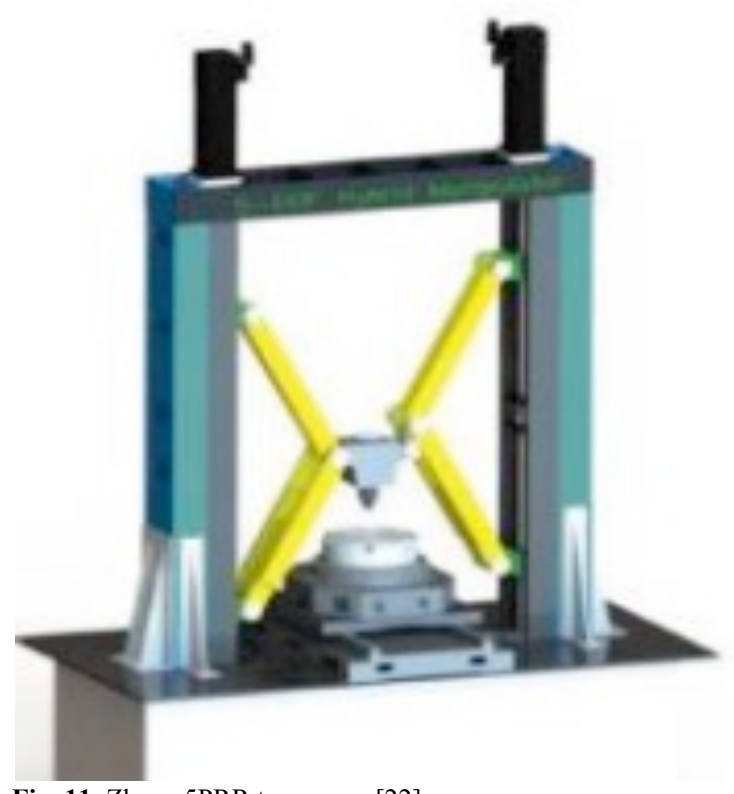

Fig. 11. Zhang 5PRR type-arms [22].

The previous PM added a rotational base in the Z-axis and could also slide through prismatic joints in the X-axis. Seward and Bonev [23] presented a 6 DOF PM focused on AM and called Hexapteron, part of the so-called Multipteron family. This PM consists of 6 arms, each composed of a cylindrical, a rotational, and a spherical joint, all in an orthogonal configuration as indicated in Fig. 12.

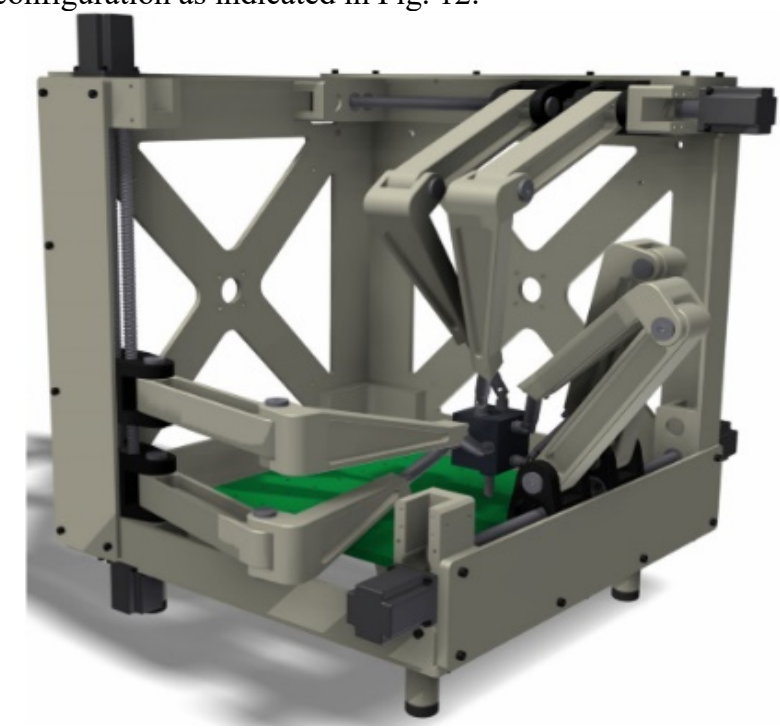

Fig. 12. Hexapteron [23]. 
Due to the structure type of the PMs, their WS is reduce compared to serial robots. To compensate for this, Viegas et al. [24] proposed a PM based on prismatic type supports attached to the arms by rotational joints. They created a structure based on five rails that form a network where the prismatic actuators can move, increasing the volume of work (Fig. 13), based on the transition between rails, which can generate eight modular assembly configurations. It was determined from the mobility analysis of the mechanism based on the sum of the number of joints, the spatiality of each arm, and the mobile platform. The robot requires only the three movements in the prismatic joints for its displacement.

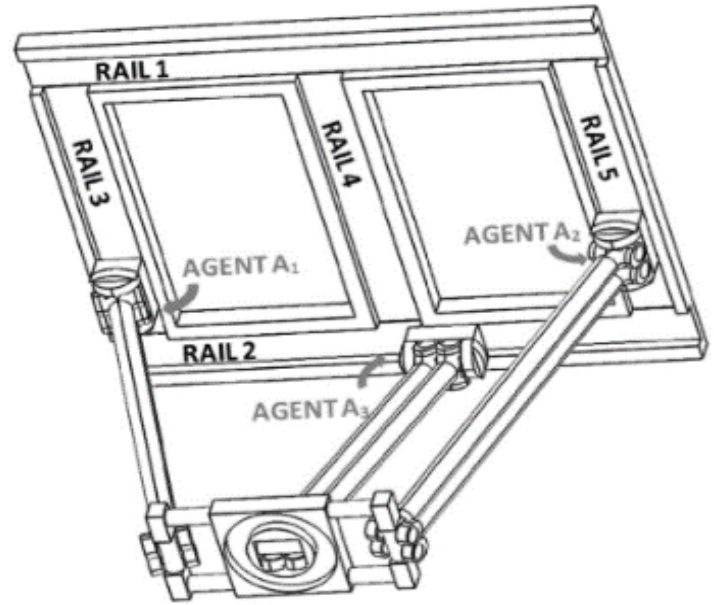

Fig. 13. Five rails PM [24].

Among other recent interesting PMs [25-31] the manipulator presented by Rong et al. [32] stands out. They proposed a configurable PM (Fig. 14) that can adapt to 32 different configurations owing to its motor locking mode, which permits many uses with different DOFs.

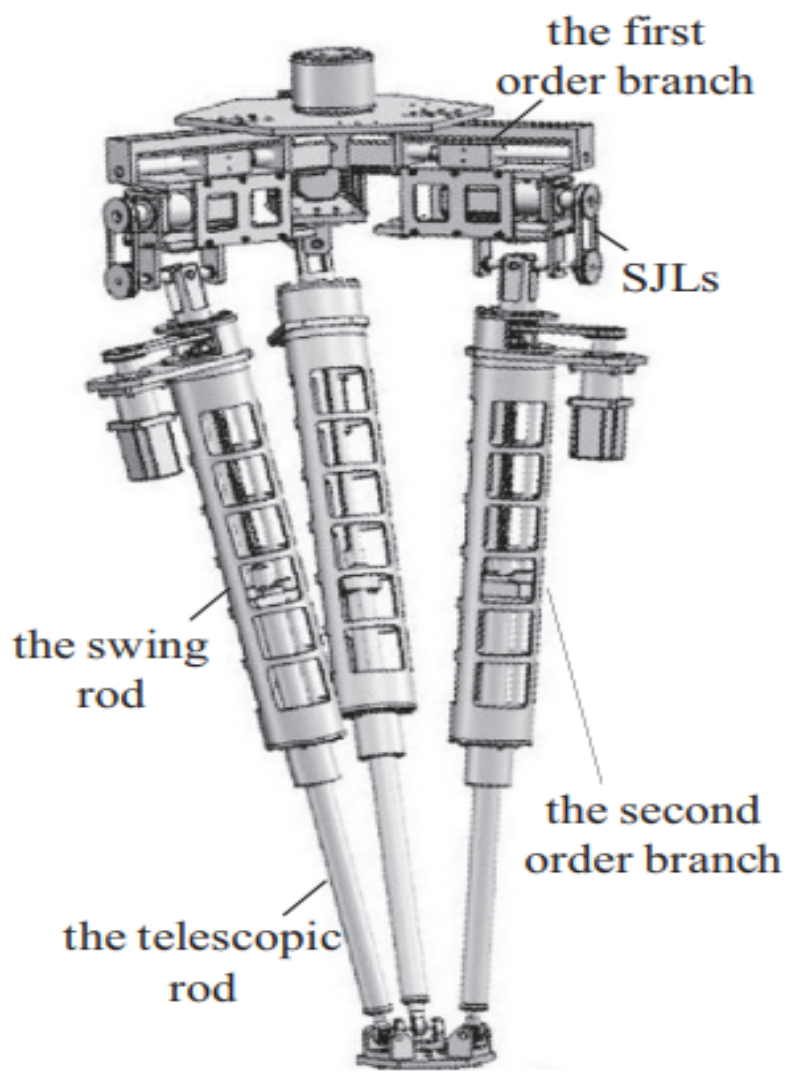

Fig. 14. Configurable PM. [32]
In the design of a PM, dynamics analysis is a crucial stage. It helps to understand the performance and serves to find the robot dimensions by optimization techniques.

\section{Dynamics}

Dynamics analysis permits the determination of the forces and torques demanded related to the movement of the mobile platform, which is required to configure the control law and the design of the PMs. There are some approaches to analyze those forces, such as Newton Euler (N-E), Euler Lagrange (E$\mathrm{L})$, virtual work (VW), among others.

Some researchers need to know the join forces and join reactions of the PMs. In these cases, the N-E formulation is appropriated. This method isolates each platform and links and determining the inertia behavior and force reaction of each one according to Newton's third law (Eq.Error! Reference source not found.)

$$
\left[\begin{array}{cc}
m I & 0 \\
0 & \zeta
\end{array}\right]\left[\begin{array}{c}
\dot{v}^{b} \\
\dot{w}^{b}
\end{array}\right]+\left[\begin{array}{c}
w^{b} \times m v^{b} \\
w^{b} \times \zeta v^{b}
\end{array}\right]=\left[\begin{array}{c}
f^{b} \\
\tau^{b}
\end{array}\right]
$$

Where $m$ is the mass of the body, $\zeta$ is the inertia tensor, $v^{b}$ and $w^{b}$ are the body velocity and angular velocity about the center of mass, respectively, $f^{b}$ and $\tau^{b}$ are the equivalence of all the forces and torques acting in the rigid body applied to the center of mass and specified for the body coordinate frame. This method works for almost all mechanisms and, on some occasions, has been validated with software simulation (i.e., ADAMS). Some evidence of this is the developments made by Hraiech et al. [33]; they worked on the dynamic's behavior of 3 DOF parallel mechanism considering its design parameters uncertainties which was determined by N-E formalism and an algorithm based on the Krawczyk operator.

Zhang, Bing, et al. [34] modeled a 3 DOF hydraulicdriven mechanism by the N-E method, and its verification was developed by software Simulink/ MATLAB and ADAMS.

Zheng et al. [35] designed and modeled a 3 DOF parallel mechanism focused on tracking trajectories. Pedrammehr et al. [36] evaluated an axis-symmetric mechanism called Hexarot, and its dynamical model was also simulated using MATLAB and ADAMS. Li, Jianfeng, et al. [37], Wang et al. [38] also considered the dynamic model of their parallel robots using the $\mathrm{N}-\mathrm{E}$ formulation.

Additionally, there are published researches done by Zhao et al.[39] and Arian et al. [40], who also work with this formulation. Even though its use is independent of the choice of coordinate system, it requires long computational times to figure out internal reactions that usually are not employed in the control law of manipulator.

$\mathrm{N}-\mathrm{E}$ method is also common in over-constrained mechanisms analysis. For instance, Bi and Kang [41] investigated the dynamic characteristics of an overconstrained PM under the N-E formulation, because of the redundant constraints, they needed to know the forces involved in joints, and for balancing the number of equations and determining all variables, they considered the material deformation too. Arian et al. [42] also analyzed the dynamics of a 3-degree-of-freedom over-constrained PM with the N-E method, analyzing the forces and torques of all the joints. The difference between results obtained with CAD software simulations and analytical methods was slight; hence, both methods are viable in this type of analysis. Other advantages 
of this method are not only the possibility of analyzing mechanical issues such as wear or clearance of the joints, just as Chen and $\mathrm{Li}$ [43] and Chen et al. [44] did; besides, this method may be used to evaluated redundant PMs [45].

Because the N-E formulation calculates the reaction forces and torques between all moving components, the high number of equations and their complexity decrement the computational efficiency calculation; hence, it complexes a control law implementation. On the other hand, Lagrange's formulation does not focus on reaction forces, so it is more computationally efficient. The E-L equation is expressed as Eq.(.

$\tau=\frac{d}{d t}\left(\frac{\delta L}{\delta \dot{q}}\right)-\left(\frac{\delta L}{\delta q}\right)$

where,

$L=K-U$

Let $\tau$ generalized torque/force, $q$ generalized coordinates, $K$ kinetics energy, and $U$ potential energy.

Nevertheless, analyzing PMs using the E-L becomes tedious because kinematics close-loop chain derivations consider all the independent generalized coordinates which generate bulk expressions.

In spite of these inconveniences, there are a lot of studies based on E-L formulation; some examples are following.

Thomas et al. [46] evaluated the closed-form dynamic model using the E-L formulation with Lagrangian multipliers and compared it with ADAMS software results.

Kuo and Tang [47] proposed a visual servoing resolved acceleration control, which aimed to acquire the end-effector position of a 3 DOF PM and reduce computations related to E-L modeling. The visual system implementation helped regulate the accelerations of the position and the orientation angle of the end-effector by imposing a visual servo approach improving the dynamic performance and time computing.

Furthermore, Muralidharan et al. [48] studied the effects of the gain-type singularity and the configuration-space singularity following an E-L formulation of forward and inverse dynamics modeling a planar and Gough-Stewart platform.

Additionally, Bernal et al. [49] reported a procedure based on E-L formulation, the projection method, and a set of nonminimal coordinates to compute the dynamics behavior of a 6-3-PUS PM which allows to easily compute the transformation matrix required to get the minimal model. Solid Works motion and Matlab were used to validate that.

Besides, Zhu et al. [50] analyzed the effect of the lateral deformation and axial deformation of a 3-TPT PM with three flexible intermediate links, its dynamics performance, and its motion error.

On the other hand, the geometries and inertia distribution were simplified, as shown in the works of Geng et al. [51] with a Stewart and Tsai and Stamper [52] platform with a PM of $3 \mathrm{DOF}$, who also developed the equations of motion based on E-L method.

The description of the final position of the actuator and the orientation in the three axes can be determined by solving nonlinear equations, as did Azulay et al. [19]. They obtained the dynamic equation of motion by Lagrange formulation and the equivalent energy principle. On the other hand, sometimes decoupling the equations of kinematic constraints helps in the motion analysis, as did Fu et al. [9].

Because there are closed kinematic chains in PMs, the inclusion and study of passive kinematic pairs must be done.
For this purpose, applications of the infinitesimal screw theory have been implemented to simplify the kinematics analysis and to synthesize the shape of the mechanisms. An example of the application of screw theory in PMs is An et al. [53]. They analyzed the motion of a 4 DOF PM, obtaining a way to find the allowed translations and rotations. Other works are those of Ye et al. [11] and Zhang [22], who, based on the analysis of movement and force of the PM with the screw theory, determined the system restrictions.

However, the E-L method's analytical calculations are still too long and prone to significant errors. Additionally, numerical calculation time increases with the number of elements; hence this method is still computationally expensive.

The principle of virtual work (VW) is a useful tool for acquiring the static and dynamic equations of multibody systems. Unlike N-E, the VW does not consider the constraint forces, just scalar work quantities to define the dynamic equations. VW aims to derive a minimum set of equations of motion of the PMs by deleting the constraint forces.

In addition, VW implementation aims to obtain equations equal to the number of the system DOFs, thus providing a procedure for determining the dynamics equations' embedding form.

VW formulation depends on infinitesimal work of external, internal, and inertial forces as well as moments of the body, as Eq. (depicts.

$$
\begin{aligned}
& \delta W=\delta W e-\delta w i=0 \\
& {[Q e-Q i] \delta q=0} \\
& Q e=Q i \\
& \tau=Q i-Q e
\end{aligned}
$$

Where $Q i$ is vector/matrix of generalized inertial forces and moments, $Q e$ is vector/matrix of generalized external and inertial forces that act on the body, $\delta q$ is a infinitesimal displacement, and $\tau$ are the torques/forces of independent actuators.

Several examples illustrated VW as a powerful tool to model the dynamic behavior of PMs mathematically, and some researchers have validated their models with mechanical simulation software. For instance, Chai et al. [54] designed and made a 3-DOF 2PRU-UPR parallel robot, its kinematics was studied by screw theory, and for its dynamic model was used VW principle. The models above were verified using ADAMS software. The VW can also solve the relation between external and inertial forces; however, the driving forces cannot be solved in some cases because the Jacobian Matrix is not a full rank matrix. As a result, Dongyi et al. [55] modeled kinematics by screw theory and calculated the inverse Jacobian matrix via relation between velocities of moving platform, actuators, and masses. This analysis was used a combining technique between N-E and VW, and its accuracy was validated by ADAMS software. Not only Danaei et al. [56] specified the dynamic model of a PM solving its kinematic using screw theory, but also Li et al. [57] and Gallardo et al. [58] use it to reduce the number of parameters to describe the PM. Additionally, Gallardo et al. [58] worked with VW and screw theory for analyzing a 4PRUR mechanism and used ADAMS. Yang et al. [59] presented a 5-DOF hybrid PM's performance analysis using the same software. 
The power consumption is essential to assess and control for design improvement. Han et al. [60] proposed a method to measure the power consumption of 4 DOF pick-and-place robot using inverse dynamic analysis and velocities and accelerations of the mobile platform. They used MATLAB and ADAMS for validating.

Besides, Xie et al. [61] proposed a 3 DOF redundant PM analyzed with two indexes coupling effects of limbs. To do these, they formulated the dynamics model based on the D'Almbert principle and VW principle. The virtual behavior simulation was conducted in ADAMS and MATLAB. Also, Alaleh et al. [62] proposed a 4 DOF over-constrained parallel manipulator based on Schönflies motion generation that provides a larger horizontal workspace with high accuracy Zaxis. Kinematics analysis was performed based on geometrical approaches, and its dynamic performance was studied following VW. Manipulator behavior was also validated by MATLAB/SIMSCAPE, showing accurate results.

Close kinematics chains of PMs cause complexities to determine kinematics behavior, and as a result, some researchers have focused on other ways. One example was carried out by Ghasemi et al. [63], who presented the PM dynamics model derived based on the VW. They proposed a visual identification system that did not need the forward kinematic model and decreased the analytical expression. The model was also verified through Matlab/SimMechanics.

Other examples of validation using MATLAB and SimMechanics, and VW were made by Song et al. [64] and Arian et al. [65].

Some PMs with active prismatic actuators need to determine the movable plate's final position based on the length of the actuators, which generally involves an algebraic system that may have more than 40 different solutions [66]. The number of solutions can be reduced by carrying out additional restrictions. However, a closed-form solution cannot be accomplished by just knowing the length of the linear actuators. Therefore it's necessary to implement either length sensors on each limb or rotation sensors on the moving plate, and the passive limbs both increase analysis time and mechanism cost. To avoid that, Seilbel et.al. [67] proposed that just by measuring the orientation of the arms and those of the movable plate, a single solution to the direct kinematics of the PM can be obtained. To validate this, they manufactured a low-cost sensor, obtaining a satisfactory response.

Another approach related to PMs' researches is their optimization. Among many objective functions, dynamics behavior is commonly used. For instance, Lu et al. [68] optimized a 3 DOF 3 PPU mechanism focused on minimizing the load of joints angular-jerk to increase PM's useful life. One of the optimization variables was dynamics behavior which was determined by the VW method and validated with MATLAB. Feng et al. [69] established the virtual power balance and dynamics model of a 3 DOF redundantly actuated PM and optimized the driving force coordination ability, work efficiency, and smoothness of the motion through a multiobjective optimization algorithm. Yu et al. [70] designed a mechanism focused on spray printing which can be modifiable to other tasks. This system was optimized using kinematics and dynamic performance. The dynamics counterpart was determined by VW theory.

Among other studies considering PM and WS were conducted by Haiqiang et al.[71], Rong et al. [32], Zhang et al. [72], Wu et al.[73] and Zhao [74].
There are several methods for assembling PMs and determining the general coordinates as a function of the active joints. General coordinates as a function of the active joints cannot be easily expressed in an analytical way, hence numerical methods have been done to achieve such function. These methods have focused on convergence and robustness. For example, Mao et.al. [75] presented a hybrid strategy between two optimization techniques (particle swarm and differential evolution) focused on the solution of the nonlinear model that describes the direct kinematics of a PM. This strategy merged the convergence response of the particle swarm-based method and the global optimization of differential evolution. The mechanical restrictions generated an equation that expresses the position and orientation of the movable plate and with the hybrid algorithm the kinematics were solved, then the comparison against other methods was made, obtaining better results with the proposed algorithm.

\section{Workspace}

The workspace (WS) is the achievable portion in space that the mobile platform can reach. In multiple studies [76], [77] two types of WS have been considered. The first one is defined as constant orientation WCO, in which the mobile plate can be moved without rotation. The second is the total orientation WT. This defines the space in which the mobile plate can reach all the orientations. In terms of multidirectionality and processes, it is relevant to determine the PM's field of action to determine the geometry of the part to be manufactured. For all the above reasons, this issue has been studied and addressed in different ways in many prototype developments.

As mentioned above, PMs have a small WS due to those settings where instantaneous continuous motion cannot be determined. These configurations are known as singularities, and many studies have concentrated on how to avoid these critical positions [78].

When designing a PM, it is also necessary to understand the intrinsic nature of the singularities and their relationship to the kinematics and configuration of the PM. Currently, there are other researches on singularities in PMs. One of the first studies was made by Hunt [79], where singularities were defined in stationary and undefined configurations. Merlet [80] proposed applying Grassmann's geometrical theory to the study of particular configurations. Later, Gosselin and Angeles [81] defined three types of singularities in terms of the matrix range. In general, a singular position in a PM is reached when the determinant of the Jacobian matrix is zero. There are direct and inverse kinematics singularities. Since the movement capacity of the mobile plate depends on the mechanical singularities of the mechanism, it is possible to evidence the direct relation between these and the WS.

Among the different ways to find a WS, there are analytical approaches such as Gosselin [82] and Bonev [83], and approaches based on the discretization. The first may be faster and more precise, but although the second has greater computational consumption (depending on sampling refinement) and with less precision, the analysis is only done once; it can be done offline and is easier to implement.

For example, Ye et al. [11] studied the WS obtaining the limiting angles in which the mechanism has no singularities successive layers with constant separation between them were then determined. For each, a sweep was made with the condition that, when analyzing the inverse kinematics, the resulting angles did not comply with the limit values of 
singularities. Similarly, Azulay et al. [19] and An et al. [53] focused on determining singularities of the discretization of space to determine the WS boundary. Closely, Xiao-Jin et al. [84] used a semi-analytic approach to study the WS of 8PSU parallel mechanism dividing the tridimensional WS into parallel subspaces. Then, they used numerical search techniques to determine the two-dimensional subspace boundary each. The sum of each subspace created the complete WS.

Mirshekari et al. [85] compared the discretized WS of three PMs, each composed of 6 arms with rotational, universal, and spherical joints, concluding that the Hexa-type PM offers the highest WS. (Fig. 15). La Mura et al. [86] not only analyzed a discrete WS; they also generated an algorithm to make the movements within the VT smooth and thus reduce inertial errors.

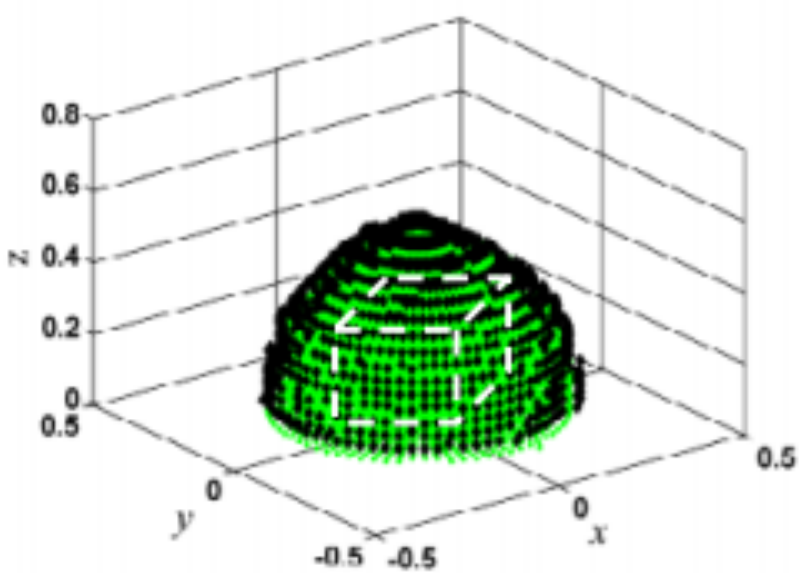

Fig. 15. Mirshekari Cloud workspace [85]

Another method for determining the VT is by solving the inverse kinematics using interval analysis. Interval analysis focuses on continuous analysis of a series of points instead of discrete points, which avoids the omission of zones related to resolution or sampling. Interval analysis provides a tool to evaluate the upper and lower limits of a function in which the intervals are unknown [87].

On the other hand, Song et al. [12] validated the motion of their PM prototype, defining the value of each joint by indicating a required location, then transformed that value into G-code. This G-code went through a mechanical constraints checker, and finally, the transformation to quaternions was done to enter the motion controller. The VW on Stewart-type platforms is restricted by two design variables: the range of motion of linear actuators and the angle of the joints.

Another WS analysis has been made based on the study of singularities when the PM has several assembly or configuration modes. Viegas et al. [24] applied the dependence of the singularities concerning the nullity of the determinants of the serial and parallel Jacobian matrices. In the development of their PM, they described that when there is a nullity in the determinant, a loss of a DOF is generated in the mobile plate, and the system becomes uncontrollable.

The basis for this was the work of Gosselin and Angeles [81]. There are other examples of WS analysis based on singularities such as Wang et al. [38].

Sometimes analyzing the position of the moving plate in non-Cartesian coordinates facilitates the work, as Filho and Cabral [14] did, who determined the WS by describing the position of the moving plate in spherical coordinates and in a function that determines whether or not restrictions are corroborating the state of the joints. For this purpose, they developed a search algorithm based on Fibonacci (Fig. 16). They described the WS with a fixed orientation of the mobile plate and the relationship to the total orientation.

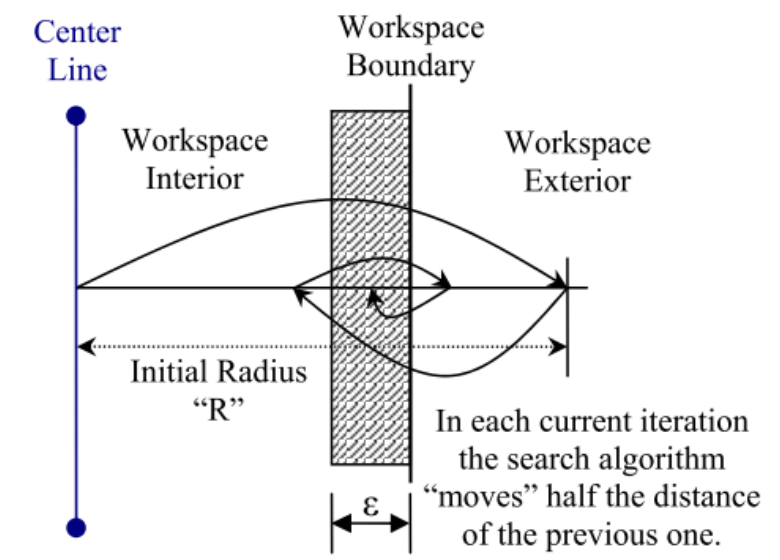

Fig. 16. Search WS boundary [14]

Sometimes, PM manufacturing is planned for a specific task, where the expected WS is known, as it was raised by Fiore et al. [88]. They defined the total WS of their PM prototype, where there were greater restrictions on the $\mathrm{X}$ and $\mathrm{Y}$ axis than on the Z-axis, similar restrictions to a Delta-type robot. For such a motive, they focused on determining a volume of work specific to form cubic, forcing the design to cover the greater possible area in $\mathrm{X}$ and $\mathrm{Y}$ without being affected by the geometric restrictions of the robot. For this, they applied genetic algorithms to find the optimal dimension of the joints.

Abeywaderna and Chen [89] also worked with predetermined volumes of work. They described the conditions under which their PM could work, determined an expected WS cylindrically (Fig. 17), and defined the dimensions of the robot. Due to the computational cost of the discretization and the expected WS axial symmetry, only one section of volumetric restrictions was analyzed. Then a circular pattern of this space was made.

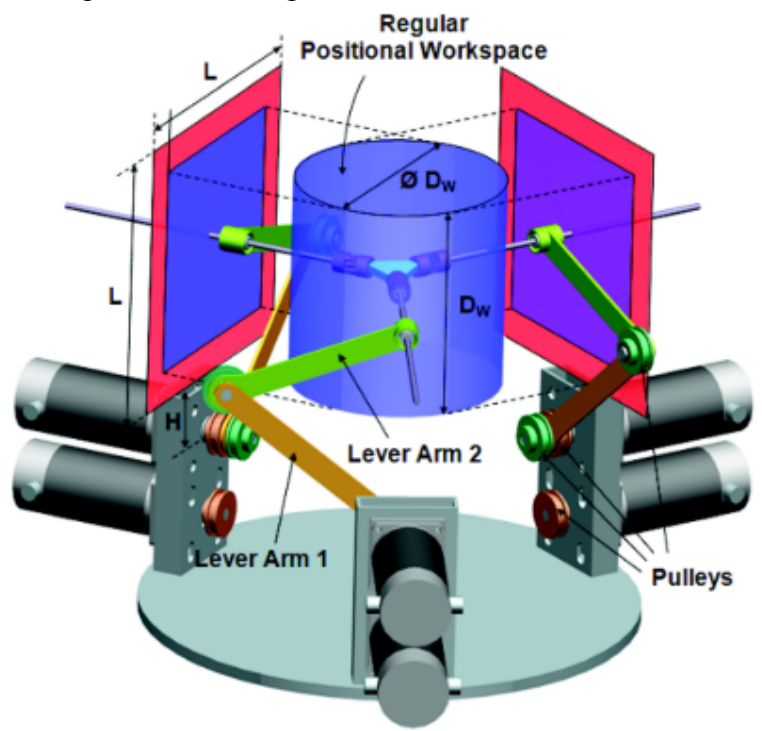

Fig. 17. Cilindrical pre-stablished WS. [89].

On other occasions, it is required to concentrate efforts on the PM movement dexterity, as Li and Angeles [18] did, who related the geometric characteristics of the robot with an index 
of dexterity, evidencing the relationship between the dimensions of the plate, dimensions of the base and the height of the mobile plate of its prototype.

Similarly, Joumah and Albitar [90] used and hybrid multiobjective method based on performance indexes for its design. Moreover, they used a numeric search method depended on the cartesian coordinate system for WS determining. They highlighted the efficiency of this method.

Yang et al. [91] carried out a multiobjective optimization using a game algorithm taking into account the reachable WS, the enclosed cylinder in that WS, and physical constraints, all of them considering polar coordinates. For spatial analysis, it has also been proposed to use dimensionless parameters to evaluate how far the configuration is from a singularity [92]. This can be useful in AM process where workspaces are shared among multiple PMs [24].

Sigger et al [93] examined singularities of a 3-RUU parallel mechanism in translational movement mode. That was achieved using algebraic constraint equations derived and the Linear Implicitization Algorithm. The method was able to calculate all input and output singularities related to WS and join space.

In the study of WSs, it is also relevant to analyze other parameters. For example, Miller [94] investigated how to design PMs in which WSs were related to manipulability and space utilization in order to optimize the field of work.

Another optimization implementation on WS adding performance indexes was proposed by Enferadi and Nikrooz [95]. This research evaluated a new kinematics and workspace behavior index named global workspace conditioning index (GWCI), which assessed the total WS reached related to roll, pitch, and yaw rotation angles around the fixed $\mathrm{x}$-axis, $\mathrm{y}$-axis, and $\mathrm{z}$-axis.

Nabavi et al. [96] presented a different concept of visual representation of WS. This concept offers data and tools for describing the orientational and translational workspace separately which helps to see maximum value of the combined motion.

From another perspective, there is the study by Zhang et.al. [22], which not only determined the WS, but also the conditional number of the Jacobian velocity matrix. This was done with a constant parallel orientation of the mobile plate with respect to the base and by modifying the angle between the mobile plate and the intermediate arm of the prototype. On the other hand, Song et al. [20] proposed to examine the WS of their prototype, defining the restrictions of the mechanism, based on the study of movement and transmissibility of global generalized virtual power, the above based on screw theory.

Because the movement of the arms is sometimes inscribed in a volume, usually a sphere, the geometric analysis of the intersections of these volumes helps to calculate the WS of a PM, usually using graphic tools, volume analysis, and computer-aided design (CAD) software, as Schreiber and Gosselin [97] did.

Another graphical way to determine the WS, Danaei et al. [98]. They introduced a novel approach to calculate a free collision WS based on a triangular intersection test using STL files. Moreover, they defined a performance index named collision-free workspace index (CFW).

Finally, one issue of PMs' mechanical behavior analysis is related to forward kinematics and its relation with WS. Because the forward kinematics is mathematically intractable, some studies use algorithms strategies to deal it. For instance, Zhang et al. [99] solved the WS including joint space of a 2RPU-2SPR PM using three back propagation neural network optimization strategies.

\section{Conclusions}

Currently, the AM is based on linear movements of the X, Y, and $\mathrm{Z}$ axes that add layer by layer of material. This type of movement generates difficulties related to the surface finish (step type finish), isotropic requirements, joining of layers, using support material (implies extra post-processing time), losing printing speeds, and the inability to print around obstacles [12] [100]. Although post-processing techniques exist, these have not solved issues caused by the unidirectionality in the Z-axis of material input. [12]. For this reason, MPs are an option to improve these drawbacks since they provide more DOF, greater stiffness, and speed of response.

In order to apply PM to AM, the mechanical structure and configuration of the robot must consider some aspects such as DOF that it provides, the volume occupied by the structure, the number and position of the actuators, among others. Some authors suggest that in AM processes, the PM doesn't need to rotate to the Z-axis, and five DOFs are enough for reducing costs [9]. Besides, it is necessary to avoid locating motors at the extremities of the arms to reduce the inertia [17]; but this ubication of motors increment the difficulty to model the mechanisms.

The methodology to find the kinematic behavior of the manipulator and its dynamics restrictions must be considered. Analytical methods have proven to be effective in simple configurations. With increasing kinematic chains the complexity increases, so other methods such as screw theory and optimization algorithms must be considered.

Because of the aim of this study is PMs implementations of AM, one must consider the dynamics behavior related to deposition process which has to be controlled. Hence, the dynamics PMs need to be modeling. There are three main principles N-E, E-L, and VW. All of them can be used to define the set of differential equations. The answer about which method is better depends on its application and computational resources. N-E provides all reactions of joints with an extensive formulation. E-L skips internal reactions but requires partial derivatives, and VW demands algebraic operations.

Another limitation of the PM is the WS; thus, it is essential to study the work capacity, mobility, manipulability, singularities, and geometrical features of the piece. It is common to work with WS by discretizing the zones and evaluating the singularities, but it is computationally costly.

If WS is part of the objective function of the optimization process, there are other suggested techniques to analyze it, such as: CAD software use [97] and cloud points study by search techniques based on optimization algorithms [14] Finally, apart from the topics covered in this writing, there are other topics related to the design and operation of PMs such as control system, optimization of the dimensions of the mechanism and control parameters through concurrent design, and the generation of path patterns deposition, all of them will be worked on in future publications.

\section{Acknowledgments}

This work was supported by Vicerrectoría de Investigaciones, Universidad Militar Nueva Granada trought project named IMP ING 3122, "Desarrollo de un sistema óptimo de deposición de material para un mecanismo de cinemática 
paralela de seis grados de libertad enfocado en manufactura aditiva". Este estudio fue realizado y soportado gracias a la Vicerrectoría de Investigaciones, Universidad Militar Nueva Granada a través del proyecto IMP ING 3122 titulado "Desarrollo de un sistema óptimo de deposición de material para un mecanismo de cinemática paralela de seis grados de libertad enfocado en manufactura aditiva".
This is an Open Access article distributed under the terms of the Creative Commons Attribution License.

\section{References}

1. Z.-F Shao, X. Tang, X. Chen, and L.P. Wang, "Research on the inertia matching of the Stewart parallel manipulator", Robot. Comput. Integr. Manuf, vol. 28 (6), pp. 649-659, 2012. DOI: https://doi.org/10.1016/j.rcim.2012.04.001.

2. P. Ogbobe, Z. Ye, H. Jiang and J. Han, "Analytical formulation of coupling effects matrix between DOF motion of parallel robots", 2010 International Conference on Intelligent Computation Technology and Automation, vol. 1, pp. 711-714, 2010. DOI: https://doi.org/10.1109/ICICTA.2010.368.

3. W. Lei, F. Qian, C. Jia and S. Liye S, "Control method of parallel robot based on adaptive neural fuzzy inference combined with PID control", Agro Food Ind. Hi. Tech, vol. 28 (3), pp. 3169-3174, 2017.

4. M. Weck and D. Staimer D, "Parallel kinematic machine tools current state and future potentials", CIRP Ann. Manuf. Technol, vol. 51 (2), pp. 671-683, 2002. DOI: https://doi.org/10.1016/S00078506(07)61706-5.

5. Y. Jiang, T. Li, L. Wang and F. Chen F "Improving tracking accuracy of a novel 3-DOF redundant planar parallel kinematic machine", Mech. Mach. Theory, vol. 119, pp. 198-218, 2018. DOI: https://doi.org/10.1016/j.mechmachtheory.2017.09.012.

6. W. Gao et al. "The status, challenges, and future of additive manufacturing in engineering", CAD Comput. Aided Des, vol. 69, pp. 65-89, 2015. DOI: https://doi.org/10.1016/j.cad.2015.04.001.

7. M. Ming, M. Kajitani and T. Higuchi, "On the design of wire parallel mechanism”, Int. J. Jpn Soc. Precis. Eng, vol. 4 (29), pp. 337-242, 1995.

8. C. Viegas, M. Tavakoli and A.T. Almeida, "A novel grid-based reconfigurable spatial parallel mechanism with large workspace", Mech. Mach. Theory, vol. 115, pp. 149-167, 2017. DOI: https://doi.org/10.1016/j.mechmachtheory.2017.05.008.

9. J. Fu, F. Gao, Y. Pan and H. Du, "Forward kinematics solutions of a special 6-degree-of-freedom parallel manipulator with three limbs", Adv. Mech. Eng, vol. 7 (5), pp. 1-11, 2015. DOI: https://doi.org/10.1177/1687814015582118

10. W. Li and J. Angeles, "Full-mobility 3- C CC parallel-kinematics machines: Forward kinematics, singularity, workspace and dexterity analyses", Mechanism and Machine Theory, vol. 126, pp. 312-328, 2018. DOI: https://doi.org/10.1016/j.mechmachtheory.2018.04.015

11. W. Ye, Y. Fang and S. Guo, "Design and analysis of a reconfigurable parallel mechanism for multidirectional additive manufacturing", Mech. Mach. Theory, vol. 112, pp. 307-326, 2017. DOI: https://doi.org/10.1016/J.MECHMACHTHEORY.2016.02.01 1.

12. X. Song, Y. Pan and Y. Chen, "Development of a low-cost parallel kinematic machine for multidirectional additive manufacturing", J. Manuf. Sci. Eng, vol. 137, pp. 1-13, 2015. DOI: https://doi.org/10.1115/1.4028897.

13. A. Dutta, D.H. Salunkhe, S. Kumar, A.D. Udai and S.V. Shah, "Sensorless full body active compliance in a 6 DOF parallel manipulator", Robotics and Computer-Integrated Manufacturing, vol. 59, pp. 278-290, 2019. DOI: https://doi.org/10.1016/j.rcim.2019.04.010.

14. S.T. Filho and E. Cabral, "Kinematics and workspace analysis of a parallel architecture robot: the Hexa", 18th Int. Congr. Mech. Eng, vol. 2, pp. 158-165, 2006.

15. C. Gosselin, T. Laliberte and A. Veillette, "Singularity-free kinematically redundant planar parallel mechanisms with unlimited rotational capability", IEEE Trans. Robot, vol. 31 (2), pp. 457-467, 2015. DOI: https://doi.org/10.1109/TRO.2015.2409433.

16. L. T. Schreiber and C. Gosselin, "Kinematically redundant planar parallel mechanisms: Kinematics, workspace and trajectory planning", Mechanism and Machine Theory, vol. 119, pp. 91-105, 2018. DOI: https://doi.org/10.1016/j.mechmachtheory.2017.08.022.

17. C. Chen, T. Gayral, S. Caro, D. Chablat, G. Moroz and S. Abeywardena, "A six degree of freedom epicyclic-parallel manipulator”, J. Mech. Robot, vol. 4 (4), pp. 041011-041018, 2012. DOI: https://doi.org/10.1115/1.4007489.

18. W. Li and J. Angeles, "The design of a 3-CPS parallel robot for maximum dexterity", Mech. Mach. Theory, vol. 122, pp. 279-291, 2018. DOI: https://doi.org/10.1016/j.mechmachtheory.2018.01.003.

19. H. Azulay, M. Mahmoodi, R. Zhao, J.K. Mills and B. Benhabib, "Comparative analysis of a new $3 \times$ PPRS parallel kinematic mechanism", Robot. Comput. Integr. Manuf, vol. 30 (4), pp. 369378, 2014. DOI: https://doi.org/10.1016/j.rcim.2013.12.003.

20. Y. Song Y, Lian B, Sun T, Dong G, Qi Y, and Gao H, "A novel fivedegree-of-freedom parallel manipulator and its kinematic optimization”, J. Mech. Robot, vol. 6 (4), pp. 041008-041016, 2014. DOI: https://doi.org/10.1115/1.4027742.

21. T. Sun and B. Lian, "Stiffness and mass optimization of parallel kinematic machine", Mech. Mach. Theory, vol. 120, pp. 73-88, 2018.

DOI: https://doi.org/doi:10.1016/j.mechmachtheory.2017.09.014.

22. D. Zhang, Y. Xu, J. Yao, B. Hu and Y. Zhao, "Kinematics, dynamics and stiffness analysis of a novel 3-DOF kinematically/actuation redundant planar parallel mechanism", Mech. Mach. Theory, vol. 116, pp. 203-219, 2017. DOI: https://doi.org/10.1016/j.mechmachtheory.2017.04.011.

23. N. Seward and I.A. Bonev, (2014) "A new 6-DOF parallel robot with simple kinematic model”, Proc. IEEE Int. Conf. Robot. Autom, pp. 4061-4066, DOI: https://doi.org/10.1109/ICRA.2014.6907449.

24. C. Viegas, M. Tavakoli and A.T. Almeida, "A novel grid-based reconfigurable spatial parallel mechanism with large workspace", Mech. Mach. Theory, vol. 115, pp. 149-167, 2017. DOI: https://doi.org/10.1016/j.mechmachtheory.2017.05.008.

25. X. Chai, M. Wang, L. Xu and W. Ye, "Dynamic Modeling and Analysis of a 2PRU-UPR Parallel Robot Based on Screw Theory", IEEE Access, vol. 8, pp. 78868-78878, 2020. DOI: https://doi.org/10.1109/ACCESS.2020.2989783.

26. S. Xie, K. Hu, H. Liu and Y. Wan, "Dynamic modeling and performance analysis of a new redundant parallel rehabilitation robot", IEEE Access 8, pp. 222211-222225, 2020. DOI: https://doi.org/10.1109/ACCESS.2020.3043429.

27. A. Arian, M. Isaksson, C. Gosselin, "Kinematic and dynamic analysis of a novel parallel kinematic Schönflies motion generator", Mechanism and Machine Theory, vol. 147, pp. 103629 1 9, 2020. DOI: https://doi.org/10.1016/j.mechmachtheory.2019.103629.

28. S. Lu, B. Ding, Y. Li, "Minimum-jerk trajectory planning pertaining to a translational 3-degree-of-freedom parallel manipulator through piecewise quintic polynomials interpolation", Advances in Mechanical Engineering, vol. 12 (3), pp. 1-18, 2020. DOI: https://doi.org/10.1177/1687814020913667.

29. H. Zhang, H. Fang, D. Zhang, Q. Zou, X. Luo, "Trajectory tracking control study of a new parallel mechanism with redundant actuation", International Journal of Aerospace Engineering, Article ID 7178103, pp. $1-14, \quad 2020$ DOI: https://doi.org/10.1155/2020/7178103.

30. G. Yu, J. Wu, L. Wang and Y. Gao, "Optimal design of the threedegree-of-freedom parallel manipulator in a spray-painting equipment", Robotica, pp. 38 (6), pp. 1064-1081, 2020. DOI: https://doi.org/10.1017/S0263574719001255.

31. H. Zhang, H. Fang, Q. Zou, D. Zhang, "Dynamic modeling and adaptive robust synchronous control of parallel robotic manipulator for industrial application", Complexity, Article ID 5640246, pp. 123, 2020. DOI: https://doi.org/10.1155/2020/5640246.

32. Y. Rong, X. Zhang, M. Qu, "Unified inverse dynamics for a novel class of metamorphic parallel mechanisms", Applied Mathematical Modelling, vol. 74 (2019), pp. 280-300, 2019. DOI: https://doi.org/10.1016/j.apm.2019.04.051. 
33. E. Hraiech, A. Chebbi, Z. Affi Z and L. Romdhane, "Error estimation and sensitivity analysis of the 3-UPU translational parallel robot due to design parameter uncertainties", Proceedings of the Institution of Mechanical Engineers, Part C: Journal of Mechanical Engineering Science, vol. 233 (8), pp. 2713-2727, 2018. DOI: https://doi.org/10.1177/0954406218793673.

34. B. Zhang B, S. Jiang, Z. Jiang, J. Li, K. Zhou and F. Liu, "Dynamic analysis and control research of a 3-dof hydraulic driven parallel mechanism", Recent Patents on Mechanical Engineering. vol. 13(2), pp. 156-170, DOI: https://doi.org/10.2174/2212797613666200210113800

35. T. Zheng, F. Zheng, X. Rui, L. Yan, K. Niu and F. Zhang, "Analysis of a three-extensible-rod tracker based on 3-RPS parallel manipulator for space large deployable paraboloid structure with power and communication integration", Acta Astronautica, vol. 169 $\begin{array}{llll}\text { (April 2020), } 2019 . & \text { pp. }\end{array}$ DOI: https://doi.org/10.1016/j.actaastro.2019.12.021.

36. S. Pedrammehr, B. Danaei, H. Abdi, M. Masouleh and S. Nahavandi, "Dynamic analysis of hexarot: axis-symmetric parallel manipulator", Robotica, vol. $36 \quad$ (2), pp. 225-240, 2018. DOI: https://doi.org/10.1017/S0263574717000315.

37. J. Li, S. Zuo, L. Zhang, M. Dong, Z. Zhang, C. Tao and R. Ji, "Mechanical design and performance analysis of a novel parallel robot for ankle rehabilitation", Journal of Mechanisms and Robotics, $\begin{array}{lllll}\text { vol. } 12 & \text { (5), } & \text { pp. } & \end{array}$ DOI: https://doi.org/10.1115/1.4046511.

38. Z. Wang, N. Zhang, X. Chai et al, "Kinematic/dynamic analysis and optimization of a 2-URR-RRU parallel manipulator", Nonlinear Dyn, vol. 88, pp. 503-519, 2017. DOI: https://doi.org/10.1007/s11071-016-3256-5.

39. Y. Zhao, H. Yu, J. Zhang, J. Yang and T. Zhao, "Kinematics, dynamics and control of a stabilized platform with a 6-RUS parallel mechanism", International Journal of Robotics and Automation, vol. 32 (3), pp. 283-290, $2017 . \quad$ DOI: https://doi.org/10.2316/Journal.206.2017.3.206-4918

40. A. Arian, B. Danaei and M.T, "Kinematics and dynamics analysis of a 2-dof spherical parallel robot", 2016 4th International Conference on Robotics and Mechatronics (ICROM), Tehran. pp. 154-159, 2016. DOI: https://doi.org/10.1109/ICRoM.2016.7886838.

41. Z.M. Bi and B. Kang, "An inverse dynamic model of overconstrained parallel kinematic machine based on newton-euler formulation”, J. Dyn. Syst. Meas. Control, vol. 136 (4), pp. 041001041009, 2014. DOI: https://doi.org/10.1115/1.4026533.

42. A. Arian, B. Danaei and M. Masouleh, "Kinematic and dynamic analysis of tripteron, an over-constrained 3-dof translational parallel manipulator, through newton-euler approach", AUT J. Model. Simul, vol. $50 \quad$ (1), pp. 61-70, 2018. DOI: https://doi.org/10.22060/MISCJ.2018.13020.5055.

43. X. Chen and Y. Li, "Dynamics analysis of spatial parallel mechanism with irregular spherical joint clearance", Shock and Vibration, vol. 2019 (5), pp.1-21, DOI: https://doi.org/10.1155/2019/6242971.

44. X. Chen, Y. Li and Y. Jia, "Dynamic response and nonlinear characteristics of spatial parallel mechanism with spherical clearance joint", Journal of Computational and Nonlinear Dynamics, vol. 14 (4), pp. 041010-041017, DOI: https://doi.org/10.1115/1.4042636.

45. B. Chang, G. Jin, "Kinematic and Dynamic Analysis of a 3PUS-S(P) Parallel Metamorphic Mechanism Used for Bionic Joint”. In: Ding X., Kong X., Dai J. (eds) Advances in Reconfigurable Mechanisms and Robots II. Mechanisms and Machine Science, vol 36. Springer, Cham, 2016. DOI: https://doi.org/10.1007/978-3-319-23327-7_41

46. M.J. Thomas, M.L. Joy and A.P. Sudheer, "Kinematic and dynamic analysis of a 3-prus spatial parallel manipulator", Chin. J. Mech. Eng, vol. 33 (13), pp. $1-17, \quad 2020$. DOI: https://doi.org/10.1186/s10033-020-0433-8.

47. Y.L. Kuo, S.C. Tang, "Dynamics and control of a 3-dof planar parallel manipulator using visual servoing resolved acceleration control", Journal of Low Frequency Noise, Vibration and Active $\begin{array}{llllll}\text { Control, vol. } 0 & (0), \quad \text { pp. } & 1-23, & 2019 .\end{array}$ DOI: https://doi.org/10.1177/1461348419876154.

48. V. Muralidharan, T.K. Mamidi, S. Guptasarma, A. Nag, S. Bandyopadhyay, "A comparative study of the configuration-space and actuator-space formulations of the Lagrangian dynamics of parallel manipulators and the effects of kinematic singularities on these", Mechanism and Machine Theory. Vol. 130, pp. 403-434, 2018. DOI: https://doi.org/10.1016/j.mechmachtheory.2018.07.009.
49. J. Bernal, R. Campa and I. Soto, "Kinematics and dynamics modeling of the 6-3-PUS-type hexapod parallel mechanism", J Mech Sci Technol, vol. 32, pp. 4555-4570, 2018. DOI: https://doiorg/10.1007/s12206-018-0901-6.

50. C. Zhu, J. Katupitiya and J. Wang, "Effect of links deformation on motion precision of parallel manipulator based on flexible dynamics", Industrial Robot, vol. 44 (6), pp. 776-787, 2017. DOI: https://doi-org.ezproxy.umng.edu.co/10.1108/IR-12-20160368.

51. Z. Geng Z, L.S. Haynes, J.D. Lee and R. Carroll, "On the dynamic model and kinematic analysis of a class of Stewart platforms", Rob. Auton. Syst. 9(4) pp. 237-254, 1992. DOI: https://doi.org/10.1016/0921-8890(92)90041-V.

52. L.W. Tsai, G.C. Walsh and R.E. Stampe, "Kinematics of a novel three DOF translational platform", Proceedings of IEEE International Conference on Robotics and Automation, vol. 4, pp. 3446-3451, DOI: https://doi.org/10.1109/ROBOT.1996.509237

53. H. An, B. Li, S. Wang and W. Ge, "Kinematics and transmission performance analyses of a $2 \mathrm{~T} 2 \mathrm{R}$ type 4-dof spatial parallel manipulator", J. Robot, vol. 2018 (Article ID 4750627), pp.1-12, 2018. DOI: https://doi.org/10.1155/2018/4750627.

54. X. Chai, M. Wang, L. Xu and W. Ye, "Dynamic modeling and analysis of a 2PRU-UPR parallel robot based on screw theory", IEEE Access, vol. 8, pp. 78868-78878, 2020. DOI: https://doi.org/10.1109/ACCESS.2020.2989783

55. L. Dongyi, L. Kun, C. Yong, Z. Wenlong, Y. Songzhu, Z. Yu, L. Junwei, Shanshuang S, "Dynamic analysis of multi-functional maintenance platform based on Newton-Euler method and improved virtual work principle", Nuclear Engineering and Technology, vol. 52 (11), p. 2020. DOI: https://doi.org/10.1016/j.net.2020.04.017.

56. Danaei B, Arian A, Masouleh M.T and Kalhor A, "Kinematic and dynamic modeling and base inertial parameters determination of the quadrupteron parallel manipulator", Computational Kinematics, pp. 249-256, 2018. DOI: https://doi.org/10.1007/978-3-319-60867928.

57. R. Li, S. Wang, D. Fan, Y. Du and S. Bai, "Dynamic modeling of a 2-RPU+2-UPS hybrid manipulator for machining application", Model. Identif. Control, vol. 38 (4), pp.169-184, 2017. DOI: https://doi.org/ 10.4173/mic.2017.4.2

58. J. Gallardo-Alvarado, R. Rodríguez-Castro and P.J. DelossantosLara, "Kinematics and dynamics of a 4-PRUR Schönflies parallel manipulator by means of screw theory and the principle of virtual work", Mech. Mach. Theory, vol. 122, pp. 347-360, 2017. DOI: https://doi.org/10.1016/j.mechmachtheory.2017.12.022

59. H. Yang, H. Fang, Y. Fang, H. Qu, "Kinematics performance and dynamics analysis of a novel parallel perfusion manipulator with passive link", Mathematical Problems in Engineering, vol. 2018 (Article ID 6768947), pp.1-18, 2018. DOI: https://doi.org/10.1155/2018/6768947.

60. G. Han, F. Xie and X.J. Liu, "Evaluation of the power consumption of a high-speed parallel robot", Front. Mech. Eng, vol. 13, pp. 167178, 2018. DOI: https://doi-org/10.1007/s11465-017-0456-8

61. S. Xie, K. Hu, H. Liu and Y. Wan, "Dynamic modeling and performance analysis of a new redundant parallel rehabilitation robot". IEEE Access, vol. 8, pp. 222211-222225, 2020. DOI: https://doi.org/10.1109/ACCESS.2020.3043429

62. A. Arian, M. Isaksso, C. Gosselin, "Kinematic and dynamic analysis of a novel parallel kinematic Schönflies motion generator", Mechanism and Machine Theory, vol. 147, pp. 103629 1-14, 2020. DOI: https://doi.org/10.1016/j.mechmachtheory.2019.103629.

63. P. Li, A. Ghasemi, W. Xie, W. Tian, "Visual closed-loop dynamic model identification of parallel robots based on optical CMM sensor", Electronics, vol. 8 (836), pp.1-23, 2019. DOI: https://doi.org/10.3390/electronics8080836.

64. X. Song, Y. Zhao, L. Jin, P. Zhang, C. Chen, "Dynamic feedforward control in decoupling space for a four-degree-of-freedom parallel robot", International Journal of Advanced Robotic Systems, vol. 2019, pp. 1-10, 2019. DOI: https://doi.org/10.1177/1729881418820451

65. A. Arian, B. Danaei, H. Abdi, S. Nahavandi, "Kinematic and dynamic analysis of the Gantry-Tau, a 3-DoF translational parallel manipulator", Applied Mathematical Modelling, vol. 51 (2017), pp.217-231, 2017. DOI: https://doi.org/10.1016/j.apm.2017.06.012.

66. P. Dietmaier P, "The stewart-gough platform of general geometry can have 40 real postures", Advances in Robot Kinematics: Analysis 


\section{Journal of Engineering Science and Technology Review 14 (4) (2021) 207 - 218}

and Control, Dordrecht, Springer, Netherlands. 1998:7-16, 1998. DOI: https://doi.org/10.1007/978-94-015-9064-81.

67. A. Seibel, S. Schulz and J. Schlattmann, "On the direct kinematics problem of parallel mechanisms", J. Robot, vol. 2018 (Article ID 2412608), pp. $1-10$, DOI: https://doi.org/10.1155/2018/2412608

68. S. Lu, B. Ding, Y. Li, "Minimum-jerk trajectory planning pertaining to a translational 3-degree-of-freedom parallel manipulator through piecewise quintic polynomials interpolation", Advances in Mechanical Engineering, vol. 12 (3), pp.1-18, 2020. DOI: https://doi.org/10.1177/1687814020913667.

69. J. Feng, T. Li, M. Han, K. Zheng and D. Yang, "Multi-objective trajectory planning method for a redundantly actuated parallel manipulator under hybrid force and position control", IEEE Access, vol. $8, \quad$ pp. $216707-216717, \quad 2020$. DOI: https://doi.org/10.1109/ACCESS.2020.3041292

70. G. Yu, J. Wu, L. Wang and Y. Gao, "Optimal design of the threedegree-of-freedom parallel manipulator in a spray-painting equipment", Robotica, vol. 38 (6), pp.1064-1081, 2020. DOI: https://doi.org/10.1017/S0263574719001255.

71. H. Zhang, H. Fang, D. Zhang, Q. Zou, X. Luo, “Trajectory tracking control study of a new parallel mechanism with redundant actuation", International Journal of Aerospace Engineering, vol. 2020 (Article ID 7178103), pp.1-14, 2020. DOI: https://doi.org/10.1155/2020/7178103.

72. H.Q. Zhang, H.R. Fang, B.S. Jiang et al. "Dynamic performance evaluation of a redundantly actuated and over-constrained parallel manipulator", Int. J Autom. Comput, vol. 16:274-285, 2019. DOI: https://doi-org/10.1007/s11633-018-1147-6.

73. J. Wu, Y. Gao, B. Zhang, L. Wang, "Workspace and dynamic performance evaluation of the parallel manipulators in a spraypainting equipment", Robotics and Computer-Integrated Manufacturing, vol. 44, pp. 199-207, 2017. DOI: https://doi.org/10.1016/j.rcim.2016.09.002.

74. Y. Zhao, Z. Zhang, G. Cheng, "Inverse rigid-body dynamic analysis for a 3UPS-PRU parallel robot", Advances in Mechanical $\begin{array}{llllll}\text { Engineering, } & \text { vol. } & 9 & \text { (2), } & \text { pp. } & 1-14,\end{array}$ DOI: https://doi.org/10.1177/1687814017693194.

75. B. Mao, Z. Xie, Y. Wang, H. Handroos and H. Wu, "A hybrid strategy of differential evolution and modified particle swarm optimization for numerical solution of a parallel manipulator. Math. Probl. Eng. 2018(Article ID 9815469) pp. 1:10, 2018. DOI: https://doi.org/ 10.1155/2018/9815469.

76. M. Daneshmand, M. Tale and M.H. Saadatzi, "Optimization of the kinematic sensitivity and the greatest continuous circle in the constant-orientation workspace of planar parallel mechanisms", International Journal of Robotics. 4(1) pp. 12-21, 2015.

77. Y. Liu, H. Wu, Y. Yang, S. Zou, X. Zhang and Y. Wang, "Symmetrical workspace of 6-ups parallel robot using tilt and torsion angles", Mathematical Problems in Engineering, vol. 2018 (Article ID 6412030), pp.1-11, DOI: https://doi.org/10.1155/2018/6412030

78. H. An, B. Li, S. Wang and W. Ge, "Kinematics and transmission performance analyses of a 2T2R type 4-dof spatial parallel manipulator", J. Robot, vol. 2018 (Article ID 4750627), pp.1-12, 2018. DOI: https://doi.org/10.1155/2018/4750627.

79. S. Parsa, R. Boudreau and J. Carretero, "Reconfigurable mass parameters to cross direct kinematic singularities in parallel manipulators", Mech. Mach. Theory, vol. 85, pp. 53-63, 2015. DOI: https://doi.org/10.1016/J.MECHMACHTHEORY.2014.10.00 8.

80. J.P. Merlet, Parallel robots, 2nd ed, Dordrecht: Springer Science \& Business Media, 2006.

81. C. Gosselin and J. Angeles, "Singularity analysis of closed-loop kinematic chains", IEEE Trans. Robot. Autom, vol. 6 (3), pp. 281290, 1990. DOI: https://doi.org/ 10.1109/70.56660.

82. C. Gosselin, "Determination of the workspace of 6-DOF parallel manipulators", J. Mech. Des, vol. 112 (3), pp.331-336, 1990. DOI: https://doi.org/10.1115/1.2912612.

83. I.A. Bonev and J. Ryu, "A geometrical method for computing the constant-orientation workspace of 6-PRRS parallel manipulators", Mech. Mach. Theory, vol. 36 (1), pp. 1-13, 2001. DOI: https://doi.org/ 10.1016/S0094-114X(00)00031-8.
84. X-J. Wan, L. Qinglei, K. Wang, "Dimensional synthesis of a robotized cell of support fixture", Robotics and Computer-Integrated Manufacturing, vol. 48, pp. 80-92, 2017. DOI: https://doi.org/10.1016/j.rcim.2017.03.001.

85. E. Mirshekari, A. Ghanbarzadeh and K.H. Shirazi, "Structure comparison and optimal design of 6-rus parallel manipulator based on kinematic and dynamic performances”, Lat. Am. J. Solids Struct, vol. 13 (13), pp. 2414-2438, 2016. DOI: https://doi.org/ $10.1590 / 1679-78252937$.

86. F. La Mura, P. Romanó, E. Fiore and H. Giberti, "Workspace limiting strategy for 6 DOF force controlled PKMs manipulating high inertia objects", Robotics, vol. 7 (1), pp.1-18, 2018. DOI: https://doi.org/ 10.3390/robotics7010010.

87. J.P. Merlet, "Interval analysis and reliability in robotics", Int. J. Reliab. Saf, vol. 3 (1), pp.104-130, 2009. DOI: https://doi.org/10.1504/IJRS.2009.026837

88. E. Fiore, H. Giberti and L. Sbaglia, "Dimensional synthesis of a 5DOF parallel kinematic manipulator for a 3D printer", 16th Int. Conf. Res. Educ. Mechatronics, REM 2015 Proc, vol. 2015, pp. 41-52, 2015. DOI: https://doi.org/10.1109/REM.2015.7380372.

89. S. Abeywardena and C. Chen, "Implementation and evaluation of a three-legged 6-degrees-of-freedom parallel mechanism as an impedance-type haptic device", IEEE/ASME Trans. Mechatronics, vol. $22 \quad$ (3), pp. 1412-1422, 2017. DOI: https://doi.org/10.1109/TMECH.2017.2682930

90. A.A. Joumah, C. Albitar, "Design optimization of 6-RUS parallel manipulator using hybrid algorithm", Int. J. Inf. Technol. Comput. $\begin{array}{llllll}\text { Sci.(IJITCS), vol. } 10 & \text { (2), } & \text { pp. } & 83-95, & 2018 .\end{array}$ DOI: https://doi.org/10.5815/ijitcs.2018.02.08

91. C. Yang, Q. Li and Q. Chen, "Multiobjective optimization of parallel manipulators using a game algorithm", Applied Mathematical Modelling, vol. 74 (2019), pp.217-243, 2019. DOI: https://doi.org/10.1016/j.apm.2019.04.058.

92. P. Grosch, R. Di Gregorio, J. Lopez and F. Thomas, "Motion planning for a novel reconfigurable parallel manipulator with lockable revolute joints", IEEE Int. Conf. Robot. Autom, vol. 2010, pp. 4697-4702, 2010. DOI: https://doi.org/10.1109/ROBOT.2010.5509305.

93. T. Stigger, M. Pfurner, M. Husty, "Workspace and singularity analysis of a 3-RUU parallel manipulator", European Conference on Mechanism Science, vol. 2018, pp. 325-332, 2018. DOI: https://doi.org/10.1007/978-3-319-98020-1_38.

94. K. Miller, "Optimal design and modeling of spatial parallel manipulators", Int. J. Rob. Res, vol. 23 (2), pp. 127-140, 2004. DOI: https://doi.org/ 10.1177/0278364904041322.

95. J. Enferadi, R. Nikrooz, "The performance indices optimization of a symmetrical fully spherical parallel mechanism for dimensional synthesis", Journal of Intelligent and Robotic Systems, vol. 90, pp. 305-321, 2017. DOI: https://doi.org/10.1007/s10846-017-0675-6.

96. S.N. Nabavi, A. Akbarzadeh, J. Enferadi, "A study on kinematics and workspace determination of a general 6-PUS robot", Journal of Intelligent and Robotic Systems, vol. 91, pp. 351-362, 2017. DOI: https://doi.org/10.1007/s10846-017-0704-5.

97. L.T. Schreiber and C. Gosselin, "Kinematically redundant planar parallel mechanisms: Kinematics, workspace and trajectory planning”, Mech. Mach. Theory, vol. 119, pp. 91-105, 2017. DOI: https://doi.org/ 10.1016/j.mechmachtheory.2017.08.022

98. B. Danaei, N. Karbasizadeh and M.T. Masouleh, "A general approach on collision-free workspace determination via triangle-totriangle intersection test", Robotics and Computer-Integrated Manufacturing, vol. 44, pp. 230-241, 2017. DOI: https://doi.org/10.1016/j.rcim.2016.08.013.

99. H. Zhang, H. Fang, D. Zhang, X. Luo and Q. Zou, "Forward kinematics and workspace determination of a novel redundantly actuated parallel manipulator", International Journal of Aerospace Engineering, vol. 2019 (Article ID 4769174), pp.1-14, 2019. DOI: https://doi.org/10.1155/2019/4769174.

100.R.J.A Allen and R.S. Trask, "An experimental demonstration of effective curved layer fused filament fabrication utilizing a parallel deposition robot", Addit. Manuf, vol. 8, pp. 78-87, 2015. DOI: https://doi.org/ 10.1016/j.addma.2015.09.001 